Microscopy

\section{Coming Events}

2018

International Microscopy Congress IMC19

September 9-14, 2018

Sydney, Australia

www.imc19.com

Fatigue Damage of Structural Materials September 16-21, 2018

Cape Cod - Hyannis, MA

www.elsevier.com/events/ conferences/international-

conference-on-fatigue-damage-of-structural-materials

\section{National Society for Histotechnology}

Symposium

September 21-26, 2018

St. Louis, MO

www.eventsinamerica.com/events/44th-annual-nsh-annualsymposium-convention-national-society-for-histotechnology/ ev57eebaa60a3d6

Structural Biology 2018: 14th International Conference on Structural Biology

September 24-26, 2018

Berlin, Germany

structuralbiology.conferenceseries.com

Microscopy: Advances, Innovation, Impact 2018

September 27, 2018

London, UK

www.rms.org.uk/discover-engage/ event-calendar/ microscopy-advances-innovation-impact-2018.htm

\section{MS\&T2018: Materials Science \&}

Technology

October 14-18, 2018

Columbus, $\mathrm{OH}$

www.matscitech.org

\section{Neuroscience 2018}

November 3-7, 2018

San Diego, CA

Sponsor: Society for Neuroscience

www.sfn.org

\section{9}

Microscopy \& Microanalysis 2019

August 4-8, 2019

Portland, OR

www.microscopy.org

\section{0}

Microscopy \& Microanalysis 2020

August 2-6, 2020

Milwaukee, WI

www.microscopy.org

\section{1}

Microscopy \& Microanalysis 2021

August 1-5, 2021

Pittsburgh, PA

www.microscopy.org

\section{2}

Microscopy \& Microanalysis 2022

July 31-August 4, 2022

Portland, OR

www.microscopy.org

\section{3}

Microscopy \& Microanalysis 2023

July 24-28, 2023

Minneapolis, MN

www.microscopy.org

More Meetings and Courses

Check the complete calendar near the

back of this magazine.

\title{
Plants Grow Up by Sensing "Down"
}

\author{
Stephen W. Carmichael \\ Mayo Clinic, Rochester, MN 55905 \\ carmichael.stephen@mayo.edu
}

The gravity sensor of plants is unique among biological sensors in its ability to combine sensitivity and robustness to cope with a fluctuating and noisy environment. It is found in specific cells, called statocytes, in which tiny assemblies of starch-rich particles, called statoliths, settle at the bottom of the cell and indicate the direction of gravity. When a shoot or root is tilted, the change in direction is detected by the statoliths within statocytes, which triggers a complex signaling pathway involving the redistribution of growth hormones within the tissue. This in turn leads to differential growth of the plant organ and the bending of the organ toward vertical. A remarkable feature of this gravitropic response is that it is extremely sensitive, even to the slightest tilt. This is key to maintaining the vertical posture of a plant on Earth.

It has long been assumed that statocytes behave as a force sensor, whereby gravity is detected by sensing the weight of statoliths on the cell edges or by interaction with the cytoskeleton. However, recent experiments indicate that the gravity sensors of plants (gravisensors) function as an inclination sensor rather than a force or acceleration sensor. This suggests that the position of the statoliths, not their weight, is the relevant gravitropic stimulus. In order to determine how this works Antoine Bérut, Hugo Chauvet, Valérie Legué, Bruno Moulia, Olivier Poulquen, and Yoël Forterre [1] conducted a series of experiments. One problem to be solved was that an assembly of particles such as sand does not respond to slight changes in tilt. A pile of grains remains static due to friction and other factors that keep the grains interlocked until a critical angle, the "avalanche angle," is reached. The sensitivity of the gravisensor in plants is not compatible with this.

Bérut et al. addressed this issue by investigating in situ the response of statolith assemblies to a wide range of inclinations over long periods of time. They found a peculiar flowing behavior not observed in classical granular material where statoliths first flow in bulk like a granular avalanche but then creep and recover a free flat surface like a liquid. To visualize statolith dynamics in response to plant inclination they examined statocytes of wheat coleoptiles. A coleoptile is the pointed protective sheath covering the emerging shoot of wheat and other plants. Unlike leaves, the pre-emergent coleoptile does not accumulate significant chlorophyll or other pigments, so it is very pale, allowing for good visualization of its interior. For this reason the wheat coleoptile

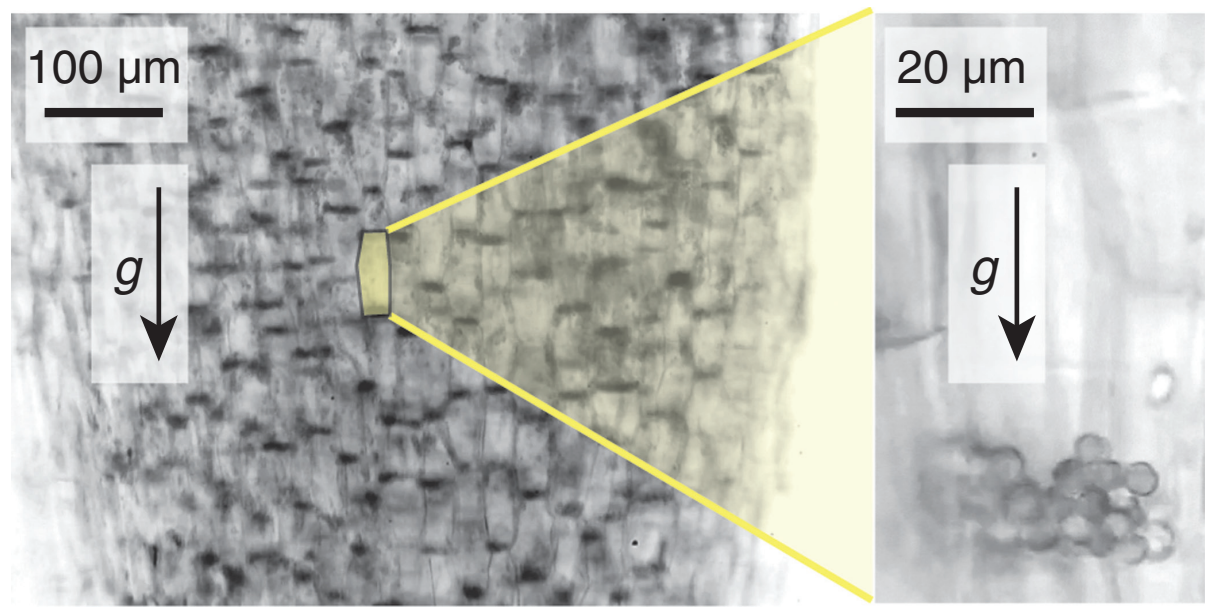

Figure 1: (Left) Visualization of statolith piles (dark areas) sedimented under gravity at the bottom of gravisensing cells in wheat coleoptiles. (Right) Close-up of a gravisesing cell showing the individual statoliths in a pile. 


\section{Sample Preparation of Nanocomposites and Nanomaterials by Uhramiorotomy}

a Powerful Alternative

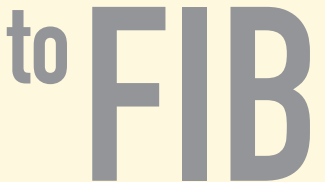

Join us at the EMS Microscopy Academy and learn the latest techniques to reveal internal structures of composites and polymers being investigated with transmission electron microscopy (TEM) and scanning transmission electron microscopy (STEM).

Sample preparation workflow will be illustrated using the Leica EM UC7 Ultramicrotome, its EM FC7 Cryochamber, and the RMC PowerTome Ultramicrotome. Differences between FIB (Focussed Ion Beam) and ultramicrotomy samples will also be covered.

\section{Who can benefit from this alternative?}

Composite and polymer research companies - especially from the automotive and aviation industries

Materials scientists already working with ultramicrotomy

FIB users preparing TEM lamellas

For more information, or to sign up for a workshop, please visit our website...

\section{www.emsdiasum.com}

DIATOME U.S.

P.O. Box $550 \cdot 1560$ Industry Rd.

Hatfield, Pa 19440

Tel: (215) 412-8390

Fax: (215) 412-8450

email: sgkcck@aol.com

\section{EMS Microscopy Academy}

P. . Box $550 \bullet 1560$ Industry Rd. • Hattield, Pa 19440 Tel: (215) 412-8400 • Fax: (215) 412-8450 Toll Free: 800-523-5874 • email: sgkcck@aol.com
Equipment used in this Technique...

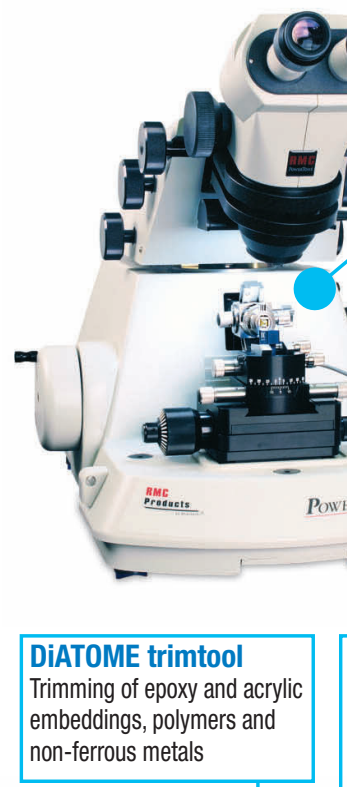

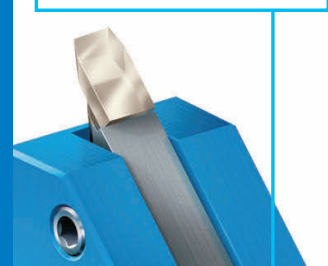

Q

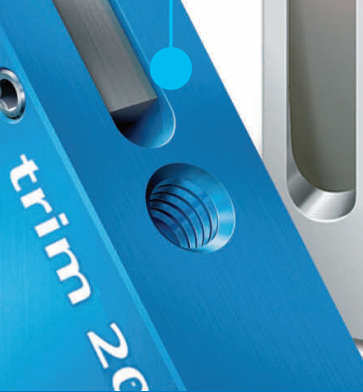

Applications...

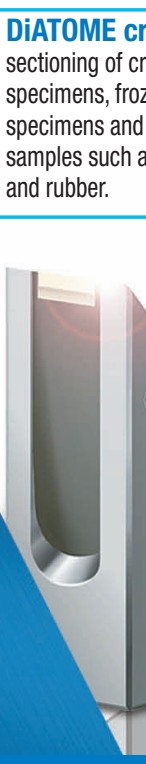

cryo
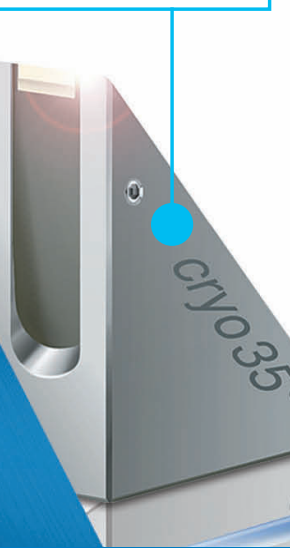

DiATOME ultra sonic Rigid polymers such as PS, PMMA, ABS, HIPS, modified PP, etc.
Zeolite USY30 Crystal morphology STEM analysis

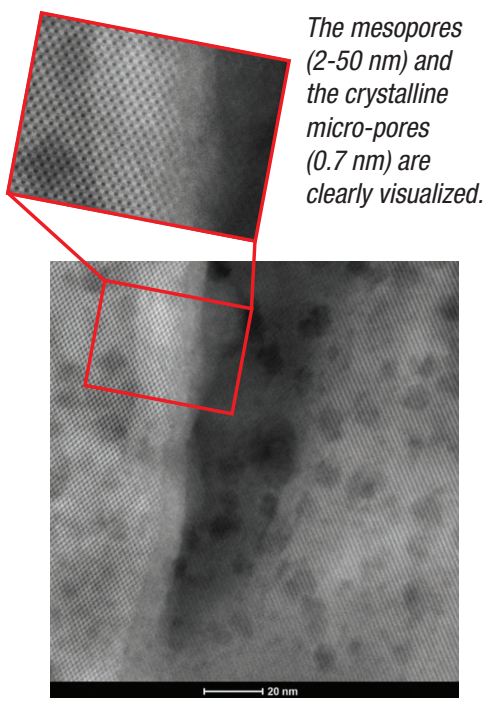

[110] Tom Willhammar, Sara Bals, EMAT Antwerpen
Epoxy loaded with amino-functionalized CNTs TEM analysis

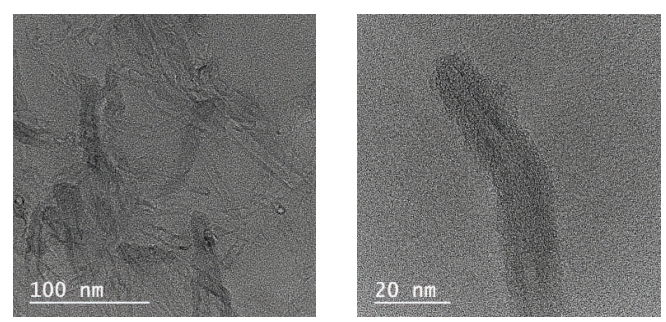

Good preservation of the interphase

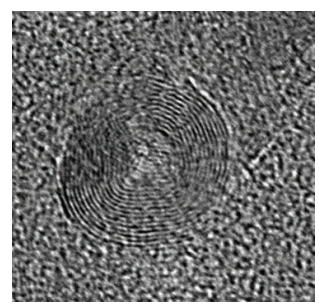


is a classical model in plant gravitropism studies. Bérut et al. laid a light microscope on its side so that the stage was oriented in the vertical plane. Sections of coleoptiles were placed vertically on the stage which could be rotated. At low magnification, groups of statoliths were seen to sediment at the bottom of cells after several minutes at rest (Figure 1, left). Higher magnification revealed that a single cell contained groups of a few tens of statoliths (about $4.5 \mu \mathrm{m}$ in diameter) in a pile at the bottom of the statocyte (Figure 1, right). Their data showed that the statoliths moved and responded to the slighted inclination of the piles. This is characteristic of a liquid, not a pile of particles.

This uncharacteristic behavior could be explained by one of two mechanisms: a physical one involving Brownian motion, or a physiological one involving the cytoskeleton. Using an in vitro system, Bérut et al. determined that Brownian motion would be effective only at a much higher temperature than normally occurs in plants. A better explanation appears to be physiological agitation, apparently by the actin-myosin network dynamics that "unlock" statoliths, allowing them to respond to any inclination. Future studies with agents or mutants that perturb the cytoskeleton could precisely explain this vital physiological process.

\section{References}

[1] A Bérut et al., PNAS Latest Articles, 2018, www.pnas.org/ doi/10.1073/pnas.1801895115.

[2] The author gratefully acknowledges Dr. Yoël Forterre for reviewing this article.

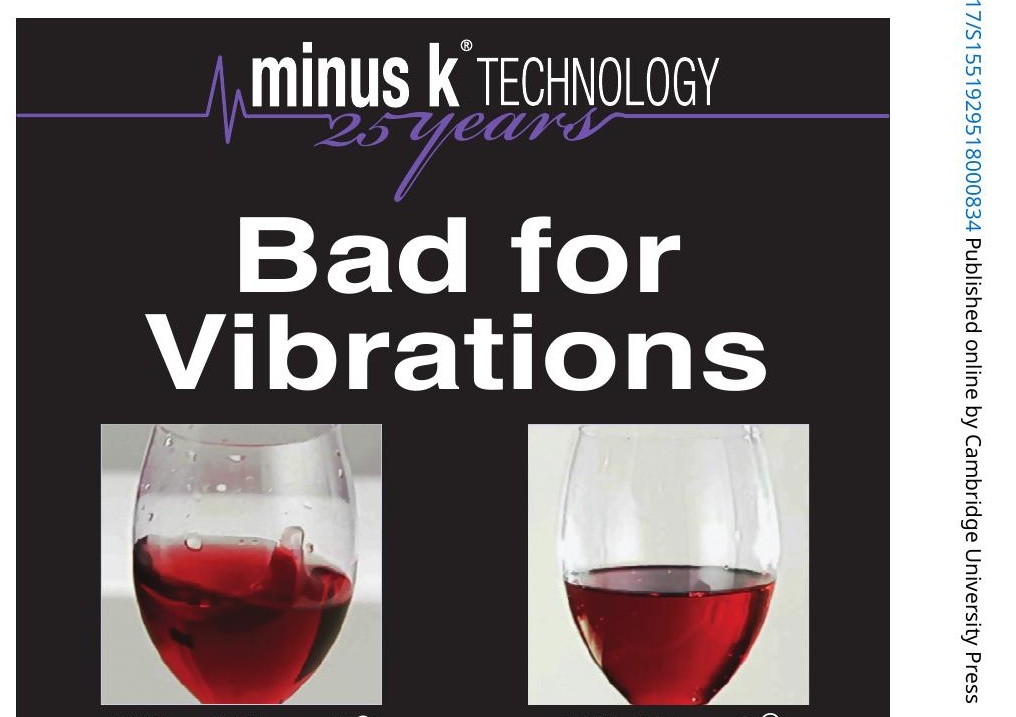

Without Minus $\mathbf{K}^{\circledR}$

With Minus $\mathbf{K}^{\circledR}$

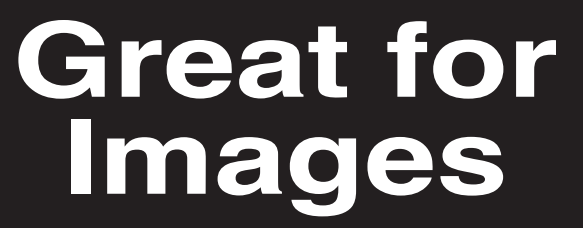

www.minusk.com

Vibration Isolation Products

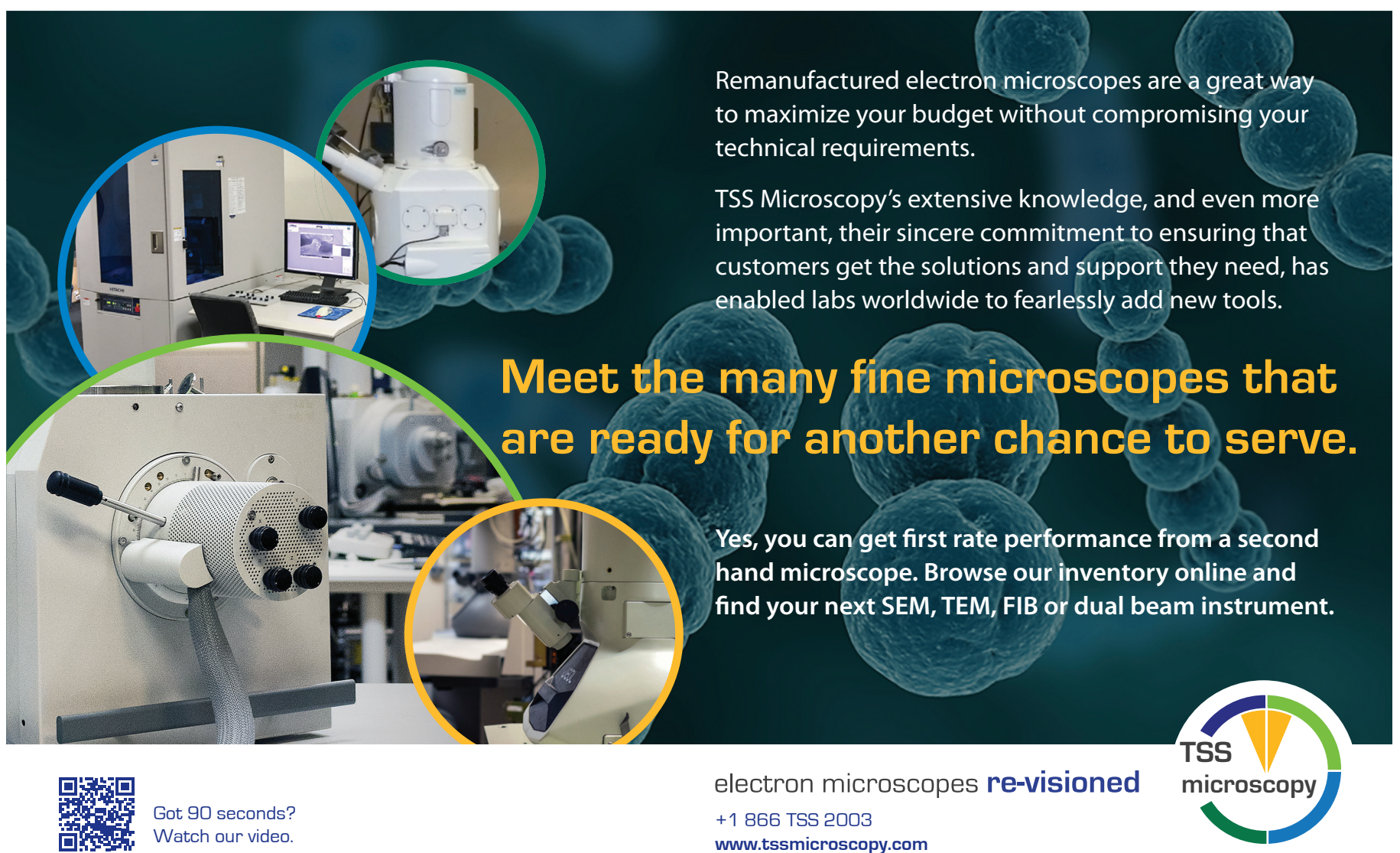




\section{Introducing}

\section{TESCAN S9000X}

Ultimate resolution and maximum throughput in large-scale sample preparation and characterisation

Extremely large cross-sections: $\mathrm{New}$ iFIB ${ }^{T M} \mathrm{Xe}^{+}$ plasma FIB with high currents and unmatched FoV redefines conventional large-area cross-sectioning and slashes sample preparation time

\section{Unveiling the most hidden features: Next} generation Triglav ${ }^{T M}$ UHR SEM column with improved and optimised in-beam detection system and extended imaging capabilities now including energy-filtering BSE signal collection for ultimate surface sensitivity

Challenging becomes routine: New Essence ${ }^{T M}$ SW GUI for effortless, faster, and smoother operations, including collision model and customisable, application-oriented layout 